\title{
The Pharmacodynamic Study of Qin Bing Eye Drop on Photokeratoconjunctivitis
}

\author{
Ruifang Xie ${ }^{1}$, Qiuhua Zhao ${ }^{2}$, Zhicheng $\mathrm{Li}^{3}$, Xin Zhou ${ }^{1 \#}$ \\ ${ }^{1}$ Department of Pharmacy, Longhua Hospital, Shanghai, China; ${ }^{2}$ National Medical Department, Youjiang Medical University for \\ Nationalities, Baishe, China; ${ }^{3}$ Department of Surgery, Pudong Hospital, Shanghai, China. \\ Email: ${ }^{* 2479707904 @ q q . c o m ~}$
}

Received May $29^{\text {th }}, 2013$; revised July $12^{\text {th }}, 2013$; accepted July $26^{\text {th }}, 2013$

Copyright (C) 2013 Ruifang Xie et al. This is an open access article distributed under the Creative Commons Attribution License, which permits unrestricted use, distribution, and reproduction in any medium, provided the original work is properly cited.

\begin{abstract}
Background: Qin Bing eye drop is prepared with cortex fraxini and borneol. It has been commonly used to relieve the symptoms of photokeratoconjunctivitis (PKC), chronic conjunctivitis and keratitis for more than forty years. However, the origins of cortex fraxini are various, which may result in the unstable quality. Methods: The animal model of PKC was established using rabbit's eyes exposure to Ultraviolet (UV); levofloxaxin drop, normal saline, Qin Bing eye drops made of herbs from Hebei province and Shanxi province were respectively instilled to both eyes of rabbits; the symptoms of rabbits' eyes were observed at different time points and scores based on weights were recorded; the recovery rates of different groups were evaluated. Results: The scores of both eyes for all rabbits were above 9 points, indicating animal models for PKC were successful; the average recovery rates of therapeutic groups, including Qin Bing eye drop groups, were higher than untreated group at different time points; the slopes of recovery rate in Qin Bing eye drop (Hebei) were above Shanxi. Conclusion: PKD animal model can be established successfully; Qin Bing eye drop groups promoted the symptoms of PKD recovering quicker than untreated group. Furthermore, Qin Bing eye drop made of herbs from province Hebei had better effects than Shanxi, which was consistent with chemical results. This indicated that chemical constituents of herbs were able to reflect the effects to a certain extent.
\end{abstract}

Keywords: Qin Bing Eye Drop; Photokeratoconjunctivitis (PKC); Cortex Fraxini

\section{Introduction}

Cortex fraxini is the dried bark of fraxinus rhynchophylla Hance, fraxinus chinensis Roxb., fraxinus chinensis Roxb. var. acuminate Lingelsh. and fraxinus stylosa Lingelsh., and called "Qin pi" in Chinese. It is a commonly used Chinese herb belonging to the "heat-clearing" category according to the classification of traditional Chinese medicine (TCM) and has the efficacy of clearing heat and eliminating dampness, improving acuity of vision, etc [1]. Pharmacological researches demonstrated that it possessed anticancer, anticoagulant, antioxidant, antiallergic, antibacterial, diuretic and central nervous system protecting activities [2]. The origins of cortex fraxini are various, including province Shanxi, Sichuan, Hebei, Liaoning, et al. [3], which may lead to the unstable quality.

\footnotetext{
"Declaration of Interest: The authors declare no conflict of interest and are solely responsible for the content of this paper.

${ }^{\#}$ Corresponding author.
}

Qin Bing eye drop is prepared with cortex fraxini and borneol. This eye drop can get rid of heat and dampness, improve eyesight, relieve pain. It has been commonly used to relieve the symptoms of photokeratoconjunctivitis (PKC), chronic conjunctivitis and keratitis in our hospital for more than forty years and exhibits good therapeutic function [4].

In our previous paper, HPLC chromatographic fingerprints of cortex fraxini were established and estimated by synthetic weighing method. Herbs from different origins were compared. The results showed the synthetic scores of province Heibei were higher than Shanxi [5]. Nevertheless, whether chemical fingerprints reflect effects need to be further confirmed.

In current paper, the animal model of PKC was established by making rabbit's eyes exposure to Ultraviolet (UV). The effects of Qin Bing eye drops made of herbs from province Hebei and Shanxi were compared. The relationship between origin and effects was discussed. 


\section{Materials and Methods}

\subsection{Materials and Reagents}

The cortex fraxini of different origins and batches were purchased from Wan Shi Cheng Limited Company (Shanghai, China). Levofloxacin eye drop (Lot 00706213) and normal saline (Lot 10082406) were respectively obtained from Zhuhai United Pharmaceutical Limited Company (Zhongshan, China) and Shanghai Changzheng Fumin Jinshan Pharmaceutical Limited Company (Shanghai, China). Pentobarbital (Lot 20100312, Fruck, Germany) were also used in this experiment.

Four batches of Qin Bing eye drops (Lot 100713, 100715, 100811 and 100819) were prepared at Long hua hospital (Shanghai, China). According to the content of main ingredients and synthetic scores of HPLC fingerprints [5] two batches respectively from province Heibei (Lot100715) and Shanxi (Lot 100819) were selected for animal experiments (Table $\mathbf{1}$ ).

\subsection{Animals}

16 of New Zealand White Rabbits (1.5 - $2.5 \mathrm{~kg}$ ), male and female half, were provided from the Yingen Rabbit Room [Shanghai, China, Licensed No. SCXK (hu) 20050003] and were housed in Animal Laboratory of Fu Dan University [Shanghai, China, Licensed No. SYXK (hu) 2009-0082]. The room temperature maintained at $20^{\circ} \mathrm{C}$ - $25^{\circ} \mathrm{C}$ under a 12-h light-12-h dark cycle $(07: 00$ 19:00) and humidity were between $40 \%$ and $70 \%$. All rabbits were raised in stainless steel cages with 2 rabbits per cage. Food and water were given ad libitum. The raising conditions were in accordance with the environmental and facilities standards of experimental animals for Health Department of People's Republic of China.

\subsection{Introduction of PKC Model}

Solution of pentobarbital $(30 \mathrm{mg} / \mathrm{ml}$ dissolved in normal saline) was administrated intravenously at $30 \mathrm{mg} / \mathrm{kg}$ in order to anaesthetize a rabbit and keep its eyes open. Next, one side of this rabbit head was placed in the light box of SJ-1031A UV Detector (Hongo Corporation, Tokyo) and the eye was exposed to ultraviolet rays for 30 min, twenty centimeters away from light emission source at 90 degrees (Figure 1), then switched to the other side. Both eyes of each rabbit were radiated twice daily. The incubation period is usually two to ten hours after exposure. Next morning both eyes of rabbits were monitored at nine o'clock by two independent observers. Typical extra-ocular symptoms of rabbits were evaluated: pain, photophobia tears, discharge, conjunctiva hyperemia, and so on. The cornea was observed using YZ5F1 slit lamp microscope (ShuZhou LiuLiu Vision technological Ltd.
Table 1. The contents and synthetic scores of HPLC fingerprints in Qin Bing eye drops.

\begin{tabular}{ccccc}
\hline Batch & Origin & $\begin{array}{c}\text { Esculin } \\
(\mu \mathrm{g} / \mathrm{mg})\end{array}$ & $\begin{array}{c}\text { Esculetin } \\
(\mu \mathrm{g} / \mathrm{mg})\end{array}$ & Synthetic scores \\
\hline 100713 & Hebei & 2.009 & 0.105 & 49.13 \\
100715 & Hebei & 2.022 & 0.113 & 98.33 \\
100811 & Shanxi & 0.586 & 0.111 & 61.16 \\
100819 & Shanxi & 0.591 & 0.113 & 69.18 \\
\hline
\end{tabular}

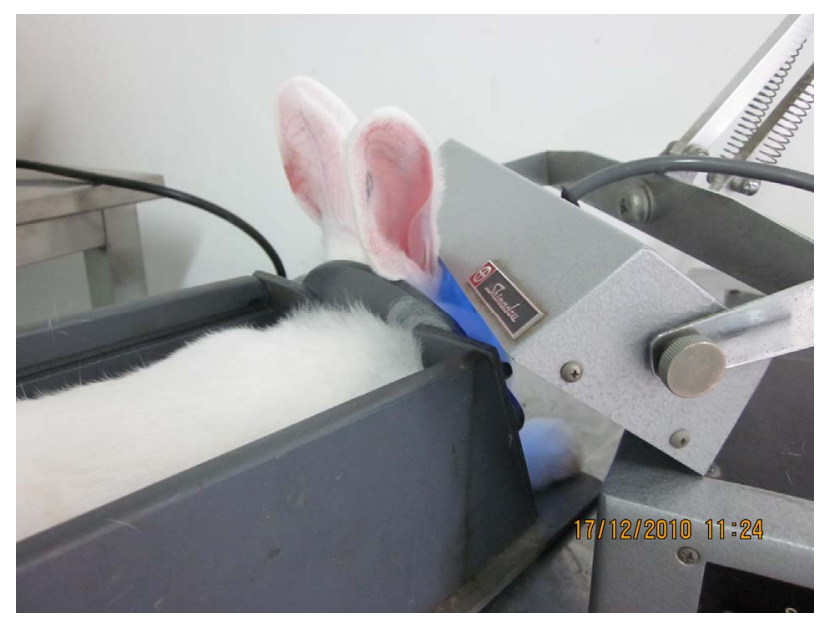

Figure 1. The electric opthalmia rabbit model by ultraviolet irradiation.

Company) with sodium fluorescein test paper (Lot 20101706, Tianjin JinMing New Technology Limited Company). Each symptom was scored as follows: 0, no change; 1 , slight; 2 , moderate; 3 , severe by a well-trained observer to minimize the variation in score. These data were recorded as vehicle.

\subsection{Administration and Assessment of Symptoms}

The rabbits were randomly divided into four group $(\mathrm{n}=$ 4): one group receiving levofloxacin eye drop as positive control, one untreated group as negative control group, and two treatment groups receiving Qin Bing eye drops (origins of cortex fraxini were respectively from Heibei and Shanxi in China). Two drops of drug solution were instilled into the right eye three times daily while the same drops of normal saline into the left eye as control until symptoms recovered completely. After first administration, symptoms of rabbits' eyes were respectively observed based on weight (Table 2) at $3 \mathrm{~h}, 6 \mathrm{~h}, 24 \mathrm{~h}, 27$ h, $30 \mathrm{~h}, 48 \mathrm{~h}, 51 \mathrm{~h}, 54 \mathrm{~h}, 72 \mathrm{~h}, 75 \mathrm{~h}$ and $78 \mathrm{~h}$.

\subsection{Statistical Analysis}

The scores of each group were represented as mean \pm SD (Standard Deviation); Recovery rate was calculated by the following equation: 


$$
\text { Recovery rate }=\frac{(\text { Scores after exposure to UV }- \text { Scores after administration })}{\text { Scores after exposure to UV }} \times 100 \%
$$

Linear regression was performed between recovery rate and time, and there was good linear relationship when the correlation coefficient was above 0.9. Data from therapeutic group and control group were evaluated by Student-test, $\mathrm{P}<0.05$ was considered statistically significant. Above analysis was carried out using Excel and SPSS 17.0 software.

\section{Results}

\subsection{Evaluation of Animal Model}

After exposure to UV for two to ten hours, eyes of rabbits exhibited the symptoms of photophobia tears, secretions and so on (Figure 2(a)). The scores of both eyes for all rabbits were above 9 points (Figure 3), indicating animal models for PKC were successful. There were no significant differences between right and left eye of rab- bits $(\mathrm{P}<0.05)$, showing these animals were appropriate for following experiment.

\subsection{Improvement of PKC Symptoms}

After instillation of eye drops, the symptoms such as photophobia tears and discharge were improved (Figures 2(b) and (c)). Thirty hours after administration, the recovery rate of each group could reach 59\% except untreated group (Table 3). The slopes of each group before and after $30 \mathrm{~h}$ showed that the symptoms recover quicker in the early stage than later period (Table 4). Furthermore, a good linear regression was observed between time and recovery rate for each group with correlation coefficient above 0.90 (Table 4), which implied that the longer period passed the better the rabbits recovered (Figure 4). After three days, the recovery rates of groups including

Table 2. Criteria for scoring ocular symptoms.

\begin{tabular}{lcccc}
\hline Ocular Parameters & \multicolumn{3}{c}{ Scores } & \\
\hline & 0 & 1 & Moderate & Severe \\
\hline Pain & Absent & Mild & Moderate & Severe \\
Photophobia tears & Absent & Mild & Moderate & Severe \\
Symptoms of conjunctiva & Absent & Mild & Moderate & Profuse \\
Discharge & Absent & Slight & $1 / 3-2 / 3$ & $>2 / 3$ \\
Corneal fluorescence staining & Negative & $<1 / 3$ & & \\
\hline
\end{tabular}

Symptoms were scored by two independent observers using the scoring system described in Supplementary. The five parameters were assessed. Each parameter was scored 0 (no disease) to 3 (maximum disease), and the sum of all five parameters represented the degree of symptoms after UV radiation and treatment. Thus, a normal eye would have a score of 0 and an eye with most severe disease would have a score of 15 ; Pain was assessed by mild pain or sense of foreign objects in eye (1), moderate pain (2), to severe pain of both eyes; Photophobia was graded as follows: 1) tears pooling at lower lid margin; 2) tears flowing over lower lid and staining eyelid; 3 ) tears staining upper and lower lids with extension below the eye socker (pesudomembranes); Symptoms of conjunctiva was assigned as follows: 0 , conjunctiva without congestion; 1 , conjunctiva mild congestion; 2 , eyelid and facial skin flushing, conjunctiva congestion; 3 , blephaospasm, blurring of vision and rainbow, significant conjunctiva congestion and edema, contracted pupil and slow reaction to light; Discharge was graded as follows: 1) discharged located to medial canthus; 2) discharge collecting along the lid margin; 3 ) profuse discharge matting fur around the eyes (and pseudomembranes); Corneal was observed using slit lamp microscope with sodium fluorescein test paper. The area of fluorescence staining was graded as $1,<1 / 3$ whereas 3 was $>1 / 3$.

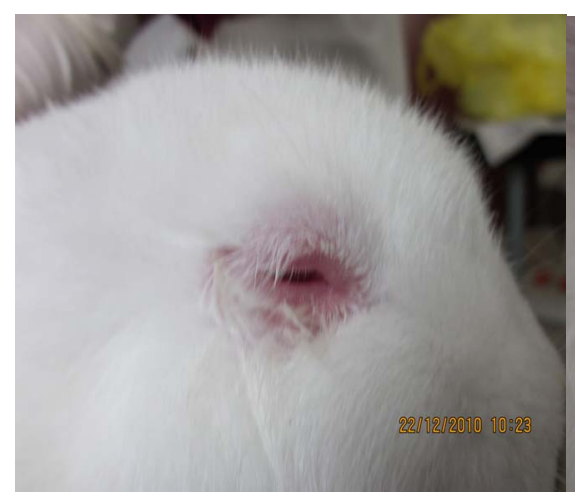

(a)

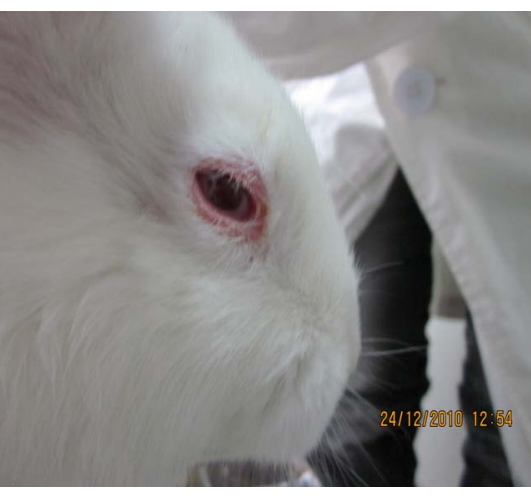

(b)

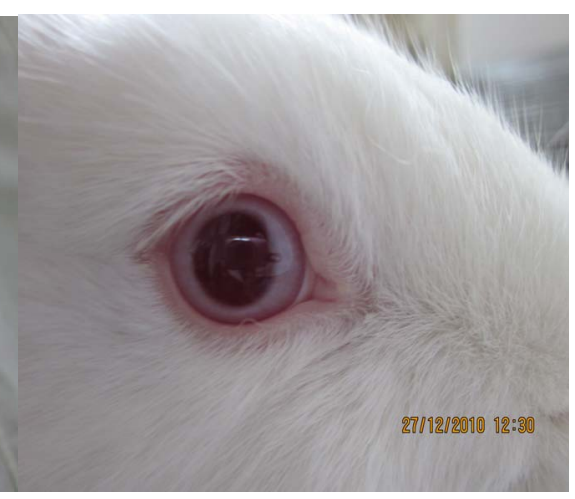

(c)

Figure 2. The alteration of symptoms for rabbit eyes: after UV radiation (a), partly relief after administration (b) and recovery completely after administration (c). 


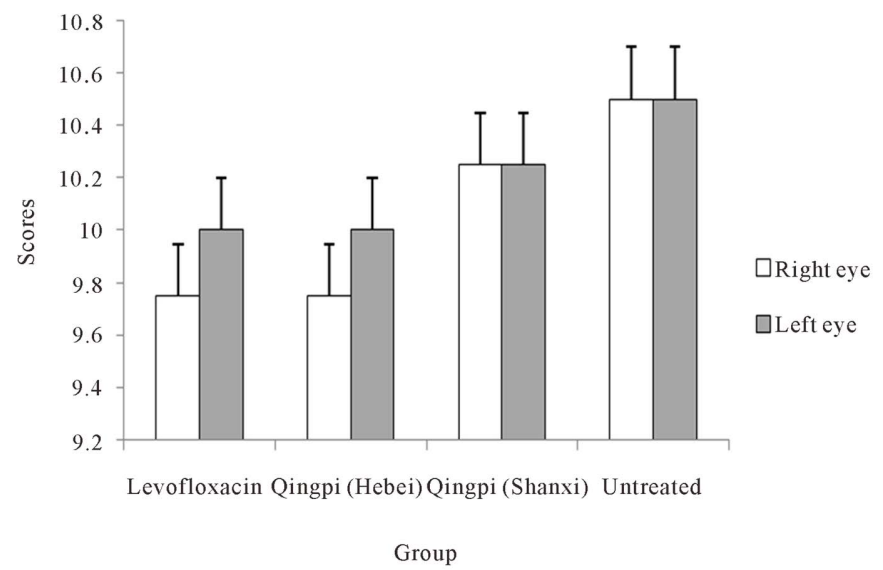

Figure 3. Scores of both eyes for animal models before administration $(n=4)$.

Table 3. Recovery rate of every group at different time points.

\begin{tabular}{cccccc}
\hline Time (h) & $\begin{array}{c}\text { Levofloxacin } \\
\text { group (\%) }(\mathrm{n}=4)\end{array}$ & $\begin{array}{c}\text { Qingbin eye drop } \\
(\text { Hebei, Lot. 100715) } \\
\text { group (\%) }(\mathrm{n}=4)\end{array}$ & $\begin{array}{c}\text { Qingbin eye drop } \\
(\text { Shanxi, Lot 100819) } \\
\text { group (\%) }(\mathrm{n}=4)\end{array}$ & $\begin{array}{c}\text { Normal saline } \\
(\%)(\mathrm{n}=16)\end{array}$ & $\begin{array}{c}\text { Untreated group } \\
(\%)(\mathrm{n}=4)\end{array}$ \\
\hline 0 & $14.77 \pm 7.01$ & $\mathbf{1 6 . 7 4} \pm \mathbf{1 1 . 1 1}$ & $9.03 \pm 6.94$ & $11.35 \pm 10.32$ & $12.50 \pm 10.75$ \\
3 & $30.78 \pm 5.70$ & $\mathbf{2 4 . 1 0} \pm \mathbf{1 2 . 4 3}$ & $22.22 \pm 3.93$ & $24.05 \pm 10.72$ & $16.67 \pm 13.61$ \\
6 & $33.90 \pm 11.29$ & $\mathbf{4 0 . 0 7} \pm \mathbf{1 5 . 9}$ & $32.29 \pm 5.24$ & $32.90 \pm 12.35$ & $25.00 \pm 11.79$ \\
24 & $51.33 \pm 9.10$ & $\mathbf{6 1 . 1 8} \pm \mathbf{4 . 6 5 ^ { * * }}$ & $45.14 \pm 13.68$ & $49.13 \pm 12.72$ & $41.67 \pm 6.80$ \\
27 & $56.53 \pm 7.56$ & $\mathbf{6 9 . 5 8} \pm \mathbf{3 . 9 4}$ & $54.17 \pm 15.96$ & $57.66 \pm 12.23$ & $52.08 \pm 10.49$ \\
30 & $64.87 \pm 16.10$ & $\mathbf{6 9 . 5 8} \pm \mathbf{3 . 9 4}$ & $59.38 \pm 11.97$ & $60.96 \pm 12.58$ & $54.17 \pm 10.76$ \\
48 & $65.06 \pm 11.42$ & $77.29 \pm 8.75$ & $79.86 \pm 4.17^{*}$ & $74.04 \pm 9.74$ & $58.33 \pm 16.67$ \\
51 & $79.17 \pm 14.43$ & $87.36 \pm 10.40$ & $88.89 \pm 10.39$ & $83.47 \pm 10.96$ & $68.75 \pm 15.77$ \\
54 & $87.50 \pm 10.21$ & $92.36 \pm 10.49$ & $88.89 \pm 10.39$ & $87.24 \pm 10.43$ & $70.83 \pm 15.96$ \\
72 & $93.75 \pm 12.50$ & $90.28 \pm 11.45$ & $96.88 \pm 6.25^{*}$ & $91.75 \pm 11.02$ & $72.92 \pm 18.48$ \\
75 & $97.92 \pm 4.17$ & $95.14 \pm 5.73$ & $96.88 \pm 6.25$ & $94.53 \pm 6.70$ & $79.17 \pm 15.96$ \\
78 & $97.92 \pm 4.17$ & $97.22 \pm 5.56$ & $100.00 \pm 0.00^{*}$ & $95.83 \pm 6.57$ & $79.17 \pm 15.96$ \\
\hline $\bar{X}$ & & & &
\end{tabular}

$\bar{X} \pm S D ; \quad{ }^{* *}$ Compared with untreated group $\mathrm{P}<0.01 ;{ }^{*}$ Compared with untreated group $\mathrm{P}<0.05$; ${ }^{*}$ The data of Normal saline group were from the data of left eyes of all the rabbits, which were instilled by normal saline.

Table 4. Regression analysis between recovery rate and time.

\begin{tabular}{lcccc}
\hline & \multicolumn{2}{c}{ Before $30 \mathrm{~h}$} & \multicolumn{2}{c}{ After $30 \mathrm{~h}$} \\
\cline { 2 - 5 } Group & Regression & Correlation coefficient & Regression & Correlation coefficient \\
\hline Levofloxacin group & $\mathrm{y}=0.0986 \mathrm{x}+0.0751$ & 0.9851 & $\mathrm{y}=0.064 \mathrm{x}+0.642$ & 0.9423 \\
Qingbin eye drop (Hebei) & $\mathrm{y}=0.1205 \mathrm{x}+0.0469$ & 0.9842 & $\mathrm{y}=0.034 \mathrm{x}+0.778$ & 0.9088 \\
Qingbin eye drop (Shanxi) & $\mathrm{y}=0.1030 \mathrm{x}+0.01$ & 0.9961 & $\mathrm{y}=0.037 \mathrm{x}+0.786$ & 0.9503 \\
Normal saline & $\mathrm{y}=0.1043 \mathrm{x}+0.0283$ & 0.9931 & $\mathrm{y}=0.041 \mathrm{x}+0.731$ & 0.9592 \\
Untreated group & $\mathrm{y}=0.0946+0.0056$ & 0.9790 & $\mathrm{y}=0.039 \mathrm{x}+0.577$ & 0.9471 \\
\hline
\end{tabular}

untreated group were above $80 \%$ (Figure 4). This indicated the symptoms of PKC could be self-healing.

\subsection{Effects Comparison of Different Groups}

For the tendency of recovery, the average recovery rates of therapeutic groups at different time points were higher than untreated group, which indicated that instillation of eye drops including normal saline promoted the symptoms of PKC (Table 3). Especially for Qing Bin eye drop groups, there were statistical differences between Qing Bin eye drop (Hebei) group and un-treatment group at 


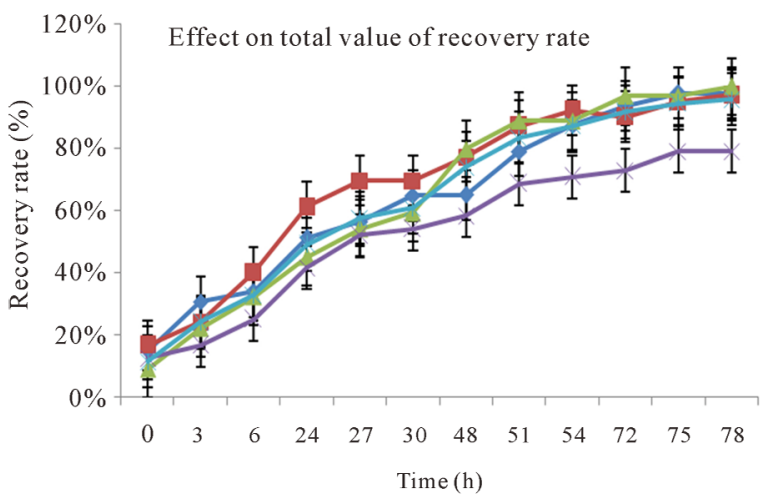

$\longrightarrow$ Levofloxacin eye drop $\rightarrow-$ Qingpi eye drop (HeBei) $\longrightarrow$ Qingpi eye drop (Shanxi) $\rightarrow$ Untreated $\quad *$ Normal Saline

Figure 4. Effects of different drugs on recovery rates $(n=$ 4).

$24 \mathrm{~h}, 27 \mathrm{~h}$ and $30 \mathrm{~h}$ as well as Qing Bin eye drop (Shanxi) group and un-treatment group at $48 \mathrm{~h}, 72 \mathrm{~h}$ and $78 \mathrm{~h}$ (Table 3). This demonstrated Qing Bin eye drops could be effective for PKC. The recovery rates of Qing Bin eye drop (Hebei) in $30 \mathrm{~h}$ were above Qing Bin eye drop (Shanxi).

For the speed of recovery (Table 4), the slopes of therapeutic groups were more than untreated group in 30 $\mathrm{h}$, which implied all the eye drops including normal saline could relieve the symptoms of PKC quicker than untreated group, especially in the early stage. Among all the eye drop groups, the slopes of Qing Bin eye drop groups in 30 hours, including Hebei and Shanxi, were higher than levofloxacin group, which suggested Qing Bin eye drops could be better than control groups. Furthermore, Qing Bin eye drop group (Hebei) was the highest $(0.1205)$. This indicated that Qingbin eye drop (Hebei) could be superior to Qing Bin eye drop (Shanxi).

\section{Discussions}

Photokeratoconjunctivitis (PKD) is caused by excessive exposure to UV radiation. Excessive UV hydrolyzes the water molecular in superficial layers of the eye to produce a large number of oxygen free radicals, which attack cells through various pathways and make cells necrosis and dropping off [6]. As a result, lots of inflammatory stimulation factors are released to induce symptoms such as ocular pain, tearing, a sense of sand in the eye and photophbia [7].

Few studies on the animal's model of electric ophthalmia have been reported. In this paper, animal model was established according to above mechanism of PKD [8]. As we known, rabbits closed eyes protectively when exposure to UV. However, they naturally opened eyes under the statue of anaesthetization. So in present experiment, intravenously anaesthetization was performed to keep animals cooperative. The injury degree depended on the intensity, distance and period of UV irradiation [9]. If the period was too long and UV intensity was too large, eyes of rabbits would be irreversibly damaged and in that case the effects of eye drops would be inconvenient to be observed. In contrast, if the degree of electric ophthamia was too mild, the rabbits would recover quickly and it was not suitable for observation. After several trials, the final parameters were optimized: the rabbits' eyes were twice exposure to both $254 \mathrm{~nm}$ and $365 \mathrm{~nm}$ of UV detector for $30 \mathrm{~min}$, twenty centimeters away from light emission source at 90 degrees. After 2 - 8 hours, the symptoms of PKD were apparent and the scores were above 9, which demonstrated the models were successful.

In most clinical cases of PKD, if no inflammation, symptoms will be self-relief in $6-8$ hours and gone within a week or so. This situation explains that the PKD symptoms of animal model are self-curative. Clinically, symptomatic treatments are commonly adopted: in early stage anaesthetic eye drops are instilled to relive pain and antibiotic eye drops or eye ointments are applied for prophylaxis of inflammation [10]. According to this routine therapeutic method, our experiment chose levofloxacin eye drop and normal saline as control medicine.

The results showed Qing Bin eye drop groups could promote the symptoms of PKD recovering quicker than untreated group. Furthermore, Qing Bin eye drop (Hebei) had better effects than Shanxi. According to our previous results (Table 1), the amount of esculin in Qing Bin eye drop (Hebei, lot. 100715) is more than that of Shanxi (lot. 100819) as well as the synthetic scores. Coumarins are proven to be the active constituents, which were reported to have activities such as anti-inflammation, antivirus, antiarthritis and anticancer [11]. Our results of effects were consistent with chemical results. This indicated that chemical constituents of herbs could reflect the effects in part.

To sum up, in this paper, the animal model of PKC was successfully established using rabbits' eyes exposure to Ultraviolet (UV). The Qin Bing eye drops made of herbs from Hebei and Shanxi were compared. Results showed that the effects of Qin Bing Eye drop (Hebei) were better than Shanxi, which was in consistence with the chemical results. This demonstrated that main constituents in cortex fraxini as marks of pharmacological effects were reasonable.

\section{Acknowledgements}

This research was supported by Longhua Medcial Project (LYTD-14) and Chinese Medicine Shanghai Health Bureau scientific research fund (No. 2008X002A).

\section{REFERENCES}

[1] National Commission of Chinese Pharmacopoeia, "Phar- 
macopoeia of Peoples Republic of China," Chemical Industry Press, Beijing, 2010, p. 254.

[2] L. J. Wang, F. Sun, X. Y. Zhang, et al., “A Secoiridoid with Quinone Reductase Inducing Activity from Cortex fraxini," Fitoterapia, Vol. 81, No. 7, 2010, pp. 834-837. doi:10.1016/j.fitote.2010.05.003

[3] X. L. Wei, C. H. Yang and J. Y. Liang, "Constituents of the Barks of Fraxinus chinensis Roxb," China Journal of National Medicine, Vol. 3, No. 4, 2005, pp. 228-230.

[4] P. Lu and M. F. Li, "Clinical Observation of 'Qinpi Eye Drop' for Chronic Conjunctivitis," Shanghai Journal of Traditional Chinese Medicine, Vol. 36, No. 9, 2002, pp. 29-31.

[5] Q. H. Zhao, X. Zhou, R. F. Xie and Z. C. Li, "Comparison of Three Weighing Methods for Evaluation of the HPLC Fingerprints of Cortex fraxini," Journal of Liquid Chromatography \& Related Technologies, Vol. 34, No. 17, 2011, pp. 2008-2019. doi:10.1080/10826076.2011.582912

[6] H. J. Han, "Clinical Analysis on 127 Cases of Photokeratoconjunctivitis," Chinese Journal of Ocular Trauma \& Occupational Eye Disease, Vol. 29, No. 4, 2007, pp.
309-310.

[7] O. Tsuyomu, O. Jun and S. Hiroyuki, "Ultraviolet Radiation Emitted by $\mathrm{CO}_{2}$ Arc Welding," The Annals of Occupational Hygiene, Vol. 45, No. 7, 2001, pp. 597-601.

[8] D. P. Zhang, S. L. Chen and S. X. Su, "The Animal Experimental Study of Fang Zhi-Ling Eye Drop on Electric Ophthalmia," Journal of Health Toxicology, Vol. 8, No. 2, 1994, pp. 105-106.

[9] Y. L. Yen, H. L. Lin, H. J. Lin, et al., "Photokeratoconjunctivitis Caused by Different Light Sources," The American Journal of Emergency Medicine, Vol. 22, No. 7, 2004, pp. 511-515. doi:10.1016/j.ajem.2004.08.003

[10] Y. Ding, Y. L. Li and G. Ma, "Integrating Treatment in Patients with Electric Ophthalmia," Chinese Journal of Today's Medicine, Vol. 4, No. 5, 2004, pp. 5-6.

[11] L. Zhou, J. Kang, F. Li, et al., "Simultaneous Analysis of Coumarins and Secoiridoids in Cortex fraxini by HighPerformance Liquid Chromatography-Diode Array Detection-Electrospray Ionization Tandem Mass Spectrometry," Journal of Pharmaceutical and Biomedical Analysis, Vol. 47, No. 1, 2008, pp. 39-46. doi:10.1016/j.jpba.2007.12.009 